\title{
CONTROLLED FUNCTIONAL DIFFERENTIAL EQUATIONS: APPROXIMATE AND EXACT ASYMPTOTIC TRACKING WITH PRESCRIBED TRANSIENT PERFORMANCE
}

\author{
Eugene P. Ryan ${ }^{1}$, Chris J. SAngwin ${ }^{2}$ and Philip Townsend ${ }^{1}$
}

\begin{abstract}
A tracking problem is considered in the context of a class $\mathcal{S}$ of multi-input, multi-output, nonlinear systems modelled by controlled functional differential equations. The class contains, as a prototype, all finite-dimensional, linear, $m$-input, $m$-output, minimum-phase systems with sign-definite "high-frequency gain". The first control objective is tracking of reference signals $r$ by the output $y$ of any system in $\mathcal{S}$ : given $\lambda \geq 0$, construct a feedback strategy which ensures that, for every $r$ (assumed bounded with essentially bounded derivative) and every system of class $\mathcal{S}$, the tracking error $e=y-r$ is such that, in the case $\lambda>0, \limsup _{t \rightarrow \infty}\|e(t)\|<\lambda$ or, in the case $\lambda=0, \lim _{t \rightarrow \infty}\|e(t)\|=0$. The second objective is guaranteed output transient performance: the error is required to evolve within a prescribed performance funnel $\mathcal{F}_{\varphi}$ (determined by a function $\varphi$ ). For suitably chosen functions $\alpha, \nu$ and $\theta$, both objectives are achieved via a control structure of the form $u(t)=-\nu(k(t)) \theta(e(t))$ with $k(t)=\alpha(\varphi(t)\|e(t)\|)$, whilst maintaining boundedness of the control and gain functions $u$ and $k$. In the case $\lambda=0$, the feedback strategy may be discontinuous: to accommodate this feature, a unifying framework of differential inclusions is adopted in the analysis of the general case $\lambda \geq 0$.
\end{abstract}

Mathematics Subject Classification. 93D15, 93C30, 34K20, 34A60.

Received June 25, 2007. Revised March 26, 2008.

Published online July 19, 2008.

\section{INTRODUCTION}

In precursors [6-8] to the present paper, an approximate tracking problem is addressed for various classes of systems. Let $\mathcal{S}$ be some given system class and let $\mathcal{R}$ be a class of reference signals. By approximate tracking, we mean attainment of the following: for any prescribed $\lambda>0$, determine a continuous output feedback strategy which ensures that, for every system (with output $y$ ) in $\mathcal{S}$ and every reference signal $r \in \mathcal{R}$, (i) the tracking error $e=y-r$ is ultimately contained in the ball of radius $\lambda$ centred at 0 (equivalently, $\limsup _{t \rightarrow \infty}\|e(t)\|<\lambda$ ), and (ii) the error $e$ exhibits prescribed transient behaviour (that is, for some suitable prescribed function $\varphi$ with $0<\liminf _{t \rightarrow \infty} \varphi(t)<\infty$, we have $\|e(t)\|<1 / \varphi(t)$ for all $\left.t>0\right)$. The present paper encompasses not only approximate tracking but also the problem of asymptotic tracking with prescribed transient behaviour: in the latter case, an output feedback strategy (possibly discontinuous) is sought which ensures that, for every system of class $\mathcal{S}$, every reference signal $r \in \mathcal{R}$ and some suitable prescribed function $\varphi$, with $\varphi(t) \rightarrow \infty$ as $t \rightarrow \infty$,

\footnotetext{
Keywords and phrases. Functional differential inclusions, transient behaviour, approximate tracking, asymptotic tracking.

${ }^{1}$ Department of Mathematical Sciences, University of Bath, Bath BA2 7AY, UK. epr@maths.bath.ac.uk;

p.townsend@bath.ac.uk

${ }^{2}$ School of Mathematics, University of Birmingham, Birmingham B15 2TT, UK. C. J.Sangwin@bham.ac.uk
} 


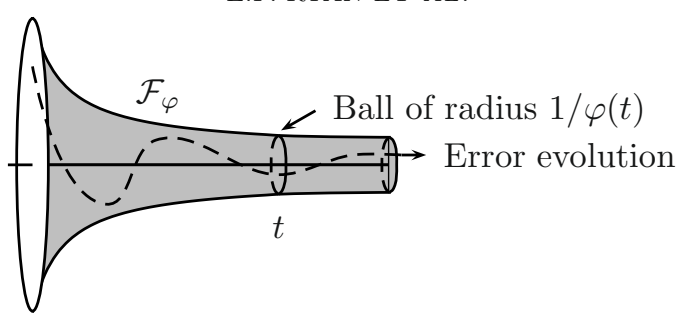

Figure 1. Performance funnel $\mathcal{F}_{\varphi}$.

we have $\|e(t)\|<1 / \varphi(t)$ for all $t>0$ (and so $e(t) \rightarrow 0$ as $t \rightarrow \infty$ ). Both cases (approximate and asymptotic tracking) are analysed within a unified framework of functional differential inclusions.

The focus of our study will be nonlinear systems (akin to those considered in [6]), with control input $t \mapsto$ $u(t) \in \mathbb{R}^{m}$, modelled by functional differential equations of the form

$$
\dot{y}(t)=f(d(t),(T y)(t), u(t)),\left.\quad y\right|_{[-h, 0]}=y^{0} \in C\left([-h, 0], \mathbb{R}^{m}\right), \quad h \geq 0,
$$

where $f$ is continuous, $T$ is a causal operator, $d$ may be thought of as a continuous and bounded perturbation, and $h \geq 0$ quantifies the "memory" of the system. As in [6-8], the class $\mathcal{R}$ of reference signals is taken to be the space $W^{1, \infty}\left(\mathbb{R}_{+}, \mathbb{R}^{m}\right)$ of bounded locally absolutely continuous functions $r: \mathbb{R}_{+} \rightarrow \mathbb{R}^{m}$ with essentially bounded derivative $\dot{r} \in L^{\infty}\left(\mathbb{R}_{+}, \mathbb{R}^{m}\right)$.

The paper is structured as follows. Section 2 formulates the control objectives and, in Section 3, a full description of the system class $\mathcal{S}$ is provided. Section 4 details the feedback structure, the potentially discontinuous nature of which leads to an interpretation of the closed-loop system in the form of a functional differential inclusion. An existence theory (which may be of independent interest) for functional differential inclusions of sufficient generality to encompass the closed-loop system is developed in Section 5 . The main results on transient behaviour and asymptotic tracking for the closed-loop system are given in Section 6 .

\section{Control objectives And the Performance Funnel}

The two control objectives are:

(i) tracking of any reference signal $r \in \mathcal{R}:=W^{1, \infty}\left(\mathbb{R}_{+}, \mathbb{R}^{m}\right)$ by the output $y$, that is, for arbitrary $\lambda \geq 0$, we seek an output feedback strategy which ensures that, for every $r \in \mathcal{R}$, every solution of the closed-loop system is bounded and the tracking error $e=y-r$ is such that either $\lim \sup _{t \rightarrow \infty}\|e(t)\|<\lambda$ if $\lambda>0$ or $\lim _{t \rightarrow \infty}\|e(t)\|=0$ if $\lambda=0$;

(ii) prescribed transient behaviour of the tracking error.

Both objectives are captured in the concept of a performance funnel

$$
\mathcal{F}_{\varphi}:=\left\{(t, e) \in \mathbb{R}_{+} \times \mathbb{R}^{m} \mid \varphi(t)\|e\|<1\right\}
$$

associated with a function $\varphi$ (the reciprocal of which determines the funnel boundary) in

$\Phi_{\lambda}:=\left\{\varphi \in A C_{\mathrm{loc}}\left(\mathbb{R}_{+}, \mathbb{R}\right) \mid \varphi(0)=0, \varphi(s)>0 \forall s>0, \liminf _{s \rightarrow \infty} \varphi(s)=1 / \lambda\right.$,

$$
\exists c>0: \quad \dot{\varphi}(s) \leq c[1+\varphi(s)] \text { for a.a. } s>0\},
$$

with the convention that, if $\lambda=0$, then $1 / \lambda:=\infty$ (and so $\varphi(t) \rightarrow \infty$ as $t \rightarrow \infty)$. Here, $A C_{\text {loc }}\left(\mathbb{R}_{+}, \mathbb{R}\right)$ denotes the space of locally absolutely continuous functions $\mathbb{R}_{+} \rightarrow \mathbb{R}$.

If a feedback structure can be devised which ensures that, for every system of the underlying class and every $r \in \mathcal{R}$, the graph of the tracking error $e=y-r$ is properly contained in $\mathcal{F}_{\varphi}$ in the sense that $\sup _{t \in \mathbb{R}_{+}} \varphi(t)\|e(t)\|<$ 1 then the tracking objective (i) is attained, and (ii) transient behaviour is governed by the choice of $\varphi$ : 
for example, if $\lambda>0$ and $\varphi$ is chosen as the function $t \mapsto \min \{t / \tau, 1\} / \lambda$, then the prescribed tracking accuracy $\lambda>0$ is achieved within the prescribed time $\tau>0$.

The intuition underpinning the feedback structure proposed below is an intrinsic high-gain property of the system class which ensures that, if $(t, e(t))$ approaches the funnel boundary, then the control input attains values sufficiently large to preclude boundary contact.

\section{Class of systems}

For $m \in \mathbb{N}$ and an interval $I \subset \mathbb{R}, C\left(I, \mathbb{R}^{m}\right)$ denotes the space of continuous functions $I \rightarrow \mathbb{R}^{m}$. If $I$ is an interval of the form $[-h, a)$ or $[-h, a], 0<a<\infty$, and $x \in C\left(I, \mathbb{R}^{m}\right)$, then, for each $\sigma \in J:=I \backslash[-h, 0)$, we define the function $x_{\sigma} \in C\left([-h, \infty), \mathbb{R}^{m}\right)$ by

$$
x_{\sigma}(t):= \begin{cases}x(t), & t \in[-h, \sigma] \\ x(\sigma), & t>\sigma .\end{cases}
$$

For $h, t \in \mathbb{R}_{+}, w \in C\left([-h, t], \mathbb{R}^{m}\right), \tau>t$ and $\delta>0$, define

$$
\mathcal{C}(w ; h, t, \tau, \delta):=\left\{v \in C\left([-h, \tau], \mathbb{R}^{m}\right)|v|_{[-h, t]}=w,\|v(s)-w(t)\| \leq \delta \quad \forall s \in[t, \tau]\right\},
$$

that is, the space of all continuous extensions $v$ of $w \in C\left([-h, t], \mathbb{R}^{m}\right)$ to the interval $[-h, \tau]$ with the property that $\|v(s)-w(t)\| \leq \delta$ for all $s \in[t, \tau]$.

We first define a class of operators $\mathcal{T}_{h}$, parameterized by $h \geq 0$.

Definition 3.1 (operator class $\mathcal{T}_{h}$ ). An operator $T$ is said to be of class $\mathcal{T}_{h}$ if, and only if, the following hold:

(i) For some $q \in \mathbb{N}, T: C\left([-h, \infty), \mathbb{R}^{m}\right) \rightarrow L_{\mathrm{loc}}^{\infty}\left(\mathbb{R}_{+}, \mathbb{R}^{q}\right)$.

(ii) $T$ is a causal operator: for all $x, y \in C\left([-h, \infty), \mathbb{R}^{m}\right)$ and all $\tau>0$

$$
x(t)=y(t) \forall t \in[-h, \tau] \Longrightarrow(T x)(t)=(T y)(t) \forall t \in[0, \tau]
$$

(iii) For each $t \geq 0$ and each $w \in C\left([-h, t], \mathbb{R}^{m}\right)$, there exist $\tau>t, \delta>0$ and $c_{0}>0$ such that

$$
\operatorname{ess-sup}_{s \in[t, \tau]}\left\|\left(T x_{\tau}\right)(s)-\left(T y_{\tau}\right)(s)\right\| \leq c_{0} \sup _{s \in[t, \tau]}\|x(s)-y(s)\| \forall x, y \in \mathcal{C}(w ; h, t, \tau, \delta) .
$$

(iv) For every $c_{1}>0$, there exists $c_{2}>0$ such that, for all $y \in C\left([-h, \infty), \mathbb{R}^{m}\right)$,

$$
\sup _{t \in[-h, \infty)}\|y(t)\| \leq c_{1} \quad \Longrightarrow \quad\|(T y)(t)\| \leq c_{2} \quad \text { for a.a. } t \geq 0 .
$$

Remark 3.2. Property (iii) is a technical assumption of local Lipschitz type which is used in establishing well-posedness of the closed-loop system (defined later in Sect. 4.1). We will have occasion to give meaning to $T x$, for a function $x \in C\left(I, \mathbb{R}^{m}\right)$ on a bounded interval $I$ of the form $[-h, a)$ or $[-h, a]$, where $0<a<\infty$. This we do by showing that $T$ "localizes", in a natural way, to an operator $\tilde{T}: C\left(I, \mathbb{R}^{m}\right) \rightarrow L_{\text {loc }}^{\infty}\left(J, \mathbb{R}^{q}\right)$, where $J:=I \backslash[-h, 0)$. In particular, and invoking causality, we may define $\tilde{T} x \in L_{\mathrm{loc}}^{\infty}\left(J, \mathbb{R}^{q}\right)$ by the property

$$
\left.\tilde{T} x\right|_{[0, \sigma]}=\left.T x_{\sigma}\right|_{[0, \sigma]} \quad \forall \sigma \in J .
$$

Henceforth, we will not distinguish notationally an operator $T$ and its "localisation" $\tilde{T}$ : the correct interpretation being clear from context. For example, with this convention in place, we may reinterpret the

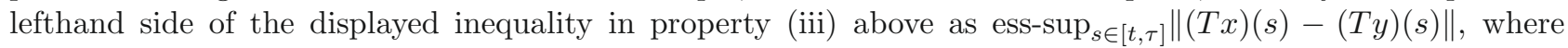
$T=\tilde{T}: C\left([-h, \tau], R^{m}\right) \rightarrow L_{\text {loc }}^{\infty}\left([0, \tau], \mathbb{R}^{q}\right)$ now represents a "localization" of the original causal operator $T: C\left([-h, \infty), \mathbb{R}^{m}\right) \rightarrow L_{\mathrm{loc}}^{\infty}\left(\mathbb{R}_{+}, \mathbb{R}^{q}\right)$. 


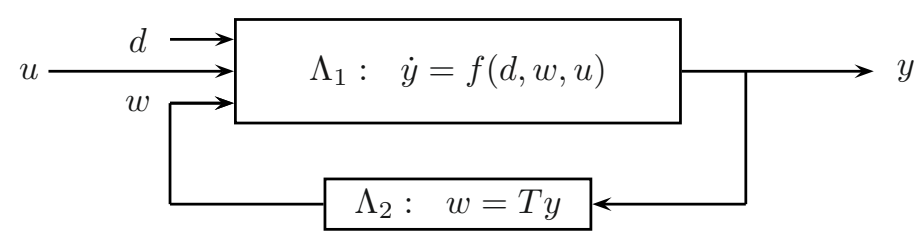

Figure 2. System of class $\mathcal{S}$.

We are now in a position to define the system class.

Definition 3.3 (system class $\mathcal{S}$ ). The class $\mathcal{S}$ is comprised of $m$-input $\left(u(t) \in \mathbb{R}^{m}\right), m$-output $\left(y(t) \in \mathbb{R}^{m}\right)$, nonlinear systems $(f, d, T)$ of the form (1.1), satisfying the following assumptions.

(A1) The function $f: \mathbb{R}^{p} \times \mathbb{R}^{q} \times \mathbb{R}^{m} \rightarrow \mathbb{R}^{m}$ is continuous.

(A2) For each compact set $\mathcal{K} \subset \mathbb{R}^{p} \times \mathbb{R}^{q}$, the continuous function $\gamma_{\mathcal{K}}: \mathbb{R} \rightarrow \mathbb{R}$, given by

$$
\gamma_{\mathcal{K}}(s):=\min \{\langle v, f(l, w, s v)\rangle \mid(l, w) \in \mathcal{K}, \quad\|v\|=1\}
$$

is such that either (i) $\lim \sup _{s \rightarrow \infty} \gamma_{\mathcal{K}}(s)=\infty$, or (ii) $\lim \sup _{s \rightarrow-\infty} \gamma_{\mathcal{K}}(s)=\infty$.

(A3) $d \in C\left(\mathbb{R}_{+}, \mathbb{R}^{p}\right)$ is bounded.

(A4) $T: C\left([-h, \infty), \mathbb{R}^{m}\right) \rightarrow L_{\mathrm{loc}}^{\infty}\left(\mathbb{R}_{+}, \mathbb{R}^{q}\right)$ is of class $\mathcal{T}_{h}$.

\subsection{Prototypical subclasses of $\mathcal{S}$}

\subsubsection{Linear prototype}

With reference to Figure 2, a system (1.1) of class $\mathcal{S}$ can be thought of as an interconnection of two subsystems. The dynamical subsystem $\Lambda_{1}$, which can be influenced directly by the control input $u$, is also driven by a disturbance $d$ and by the output $w$ from the subsystem $\Lambda_{2}$, formulated as a causal operator mapping the the signal $y$ to $w$ (an internal quantity, unavailable for feedback purposes).

To illustrate this, consider the prototype class $\mathcal{L}$ of finite-dimensional, minimum-phase, $m$-input $\left(u(t) \in \mathbb{R}^{m}\right)$, $m$-output $\left(y(t) \in \mathbb{R}^{m}\right)$ linear systems $(A, B, C)$ with sign-definite high-frequency gain, in the sense that either $C B$ or $-C B$ is positive definite (symmetry of $C B$ is not assumed). The minimum-phase property is characterized by

$$
\operatorname{det}\left[\begin{array}{cc}
s I-A & B \\
C & 0
\end{array}\right] \neq 0 \text { for all } s \in \mathbb{C}_{+}:=\{s \in \mathbb{C} \mid \operatorname{Re}(s) \geq 0\}
$$

Specifically,

$$
\mathcal{L}=\left\{(A, B, C) \mid A \in \mathbb{R}^{n \times n}, B \in \mathbb{R}^{n \times m}, C \in \mathbb{R}^{m \times n}, m, n \in \mathbb{N}, m \leq n, C B \text { sign definite, (3.2) holds }\right\} .
$$

It is well known (see for example [4], Lem. 2.1.3) that, for each $(A, B, C) \in \mathcal{L}$ (and assuming $m<n$ ), there exists a similarity transformation which takes the system into the form

$$
\left.\begin{array}{ll}
\dot{y}(t)=A_{1} y(t)+A_{2} z(t)+C B u(t), & y(0)=y^{0}, \\
\dot{z}(t)=A_{3} y(t)+A_{4} z(t), & z(0)=z^{0},
\end{array}\right\}
$$

where, by the minimum-phase property, $A_{4}$ is a Hurwitz matrix. Defining the function $d$ (continuous and bounded) and operator $T$ (linear) by

$$
d(t):=A_{2}\left(\exp \left(A_{4} t\right)\right) z^{0}, \quad(T y)(t):=A_{1} y(t)+A_{2} \int_{0}^{t}\left(\exp A_{4}(t-s)\right) A_{3} y(s) \mathrm{d} s
$$


we see that the original system $(A, B, C) \in \mathcal{L}$ can be recast in the form of the (linear) functional differential equation

$$
\dot{y}(t)=d(t)+(T y)(t)+C B u(t), \quad y(0)=y^{0} \in \mathbb{R}^{m},
$$

which is of the form (1.1) with $h=0$ and $f: \mathbb{R}^{m} \times \mathbb{R}^{m} \times \mathbb{R}^{m} \rightarrow \mathbb{R}^{m},(l, w, v) \mapsto l+w+C B v$. Clearly, Assumption (A1) holds. Since $A_{4}$ is Hurwitz, we see that (A3) and (A4) (with $h=0$ ) are valid. It remains to show that (A2) also holds. Recall that $C B$ is sign definite and so either (i) $C B$ is positive definite, which we write symbolically as $C B>0$, or (ii) $-C B>0$. Let $\mathcal{K} \subset \mathbb{R}^{m} \times \mathbb{R}^{m}$ be compact and define

$$
c_{\mathcal{K}}:=\min \{\langle v, l+w\rangle \mid(l, w) \in \mathcal{K},\|v\|=1\} .
$$

Now, observe that

$$
\begin{aligned}
C B>0 & \Longrightarrow \min \{\langle v, C B v\rangle \mid\|v\|=1\}=\frac{1}{2}\left\|\left(C B+B^{T} C^{T}\right)^{-1}\right\|^{-1} \\
-C B>0 & \Longrightarrow \min \{\langle v, C B v\rangle \mid\|v\|=1\}=-\frac{1}{2}\left\|C B+B^{T} C^{T}\right\|
\end{aligned}
$$

Therefore,

(i) $C B>0, s \geq 0 \Longrightarrow \gamma_{\mathcal{K}}(s) \geq c_{\mathcal{K}}+\frac{1}{2} s\left\|\left(C B+B^{T} C^{T}\right)^{-1}\right\|^{-1}$ and so (A2)(i) holds,

(ii) $-C B>0, s \leq 0 \Longrightarrow \gamma_{\mathcal{K}}(s) \geq c_{\mathcal{K}}-\frac{1}{2} s\left\|C B+B^{T} C^{T}\right\| \quad$ and so (A2)(ii) holds.

\subsubsection{Systems with input nonlinearity}

To illustrate the generality afforded by Assumption (A2), consider a single-input, single-output $(m=1)$ system (3.3) of class $\mathcal{L}$ with a nonlinearity $g$ in the input channel

$$
\left.\begin{array}{ll}
\dot{y}(t)=A_{1} y(t)+A_{2} z(t)+\beta g(u(t)), & y(0)=y^{0}, \\
\dot{z}(t)=A_{3} y(t)+A_{4} z(t), & z(0)=z^{0},
\end{array}\right\}
$$

where $\beta:=C B$ is now a non-zero real number. We assume only that $g: \mathbb{R} \rightarrow \mathbb{R}$ is a continuous unbounded function with bounded even part, for example, $g: v \mapsto(1+v) \cos v$. Such a function can influence/reverse the polarity of an input signal $u(\cdot)$ in a manner unpredictable by a controller. Defining $d$ and $T$ as in (3.4), system (3.5) can be expressed as

$$
\dot{y}(t)=d(t)+(T y)(t)+\beta g(u(t)), \quad y(0)=y^{0} \in \mathbb{R},
$$

which again is of form (1.1). Assumptions (A1), (A3) and (A4) clearly hold. Define $g_{o}$ and $g_{e}$ to be the odd and even parts, respectively, of the function $\beta g$. To see that (A2) holds, let $\mathcal{K} \subset \mathbb{R} \times \mathbb{R}$ be compact, define $c_{\mathcal{K}}$ as above, and observe that, since $v g_{o}(s v)=g_{o}(s)$ for all $|v|=1$ and all $s \in \mathbb{R}$,

$$
\gamma_{\mathcal{K}}(s)=\min \left\{v\left(l+w+g_{e}(s v)\right)|(l, w) \in \mathcal{K},| v \mid=1\right\}+g_{o}(s) \geq c_{\mathcal{K}}-\left|g_{e}(s)\right|+g_{o}(s) \quad \forall s .
$$

Since the function $g_{o}$ is odd and unbounded, there must exist an unbounded monotone sequence $\left(s_{n}\right)$ (either strictly increasing or strictly decreasing) such that $g_{o}\left(s_{n}\right) \rightarrow \infty$ as $n \rightarrow \infty$ which, together with boundedness of $g_{e}$ and (3.6), ensures $\gamma_{\mathcal{K}}\left(s_{n}\right) \rightarrow \infty$ as $n \rightarrow \infty$.

\subsubsection{Nonlinear systems}

Now consider a further generalization of systems of form (3.5) to nonlinear systems of the form

$$
\left.\begin{array}{ll}
\dot{y}(t)=f_{1}(y(t), z(t))+g(u(t)), & y(0)=y^{0} \in \mathbb{R}, \\
\dot{z}(t)=f_{2}(y(t), z(t)), & z(0)=z^{0} \in \mathbb{R}^{p},
\end{array}\right\}
$$


with $f_{1}$ continuous, $f_{2}$ locally Lipschitz, and (as above) $g$ continuous and unbounded with bounded even part (we have absorbed the parameter $\beta \neq 0$ in $g$ ). Temporarily regarding $y$ as an independent input to the second subsystem in (3.7), denote the unique solution of the initial-value problem $\dot{z}=f_{2}(y, z), z(0)=z^{0}$, by $z\left(\cdot ; z^{0}, y\right)$. If we now assume that the second subsystem in (3.7) is input-to-state stable (ISS) (see [13]), then, for each $z^{0} \in \mathbb{R}^{p}$, we may define an operator $C\left(\mathbb{R}_{+}, \mathbb{R}\right) \rightarrow C\left(\mathbb{R}_{+}, \mathbb{R} \times \mathbb{R}^{p}\right)$ by

$$
(T y)(t):=\left(y(t), z\left(t ; z^{0}, y\right)\right) \quad \forall t \in \mathbb{R}_{+} .
$$

This operator $T$ is of class $\mathcal{T}_{0}$ (Assumption (A4) holds with $h=0, m=1$ and $q=p+1$ ). System (3.7) may be expressed as the functional differential equation

$$
\dot{y}(t)=f_{1}((T y)(t))+g(u(t)), \quad y(0)=y^{0},
$$

which is of the form (1.1) with $h=0$ and $f:(l, w, v) \mapsto f_{1}(w)+g(v)$. Evidently, Assumption (A1) holds, Assumption (A3) is vacuous, and Assumption (A2) holds by the argument (mutatis mutandis) used in Section 3.1.2.

\subsubsection{Systems with delays and hysteresis}

Finally, we remark that nonlinear delay elements are incorporated in the operator class $\mathcal{T}_{h}$, see for example [12], whilst the class $\mathcal{T}_{0}$ encompasses a wide range of hysteresis operators, including many physically motivated effects: as observed in [5], examples such as relay hysteresis, elastic-plastic hysteresis, backlash hysteresis, Prandtl and Preisach operators (for background, see $[2,10]$ ) are of class $\mathcal{I}_{0}$.

\section{FeEdBack CONTROL}

We proceed to make precise the proposed output feedback structure. Let $\lambda \geq 0$ and $\varphi \in \Phi_{\lambda}$. Let $\nu: \mathbb{R} \rightarrow \mathbb{R}$ be any continuous function with the properties

$$
\limsup _{k \rightarrow \infty} \nu(k)=+\infty \quad \text { and } \quad \liminf _{k \rightarrow \infty} \nu(k)=-\infty,
$$

for example, $\nu: k \mapsto k \cos k$. Let $\alpha:[0,1) \rightarrow \mathbb{R}_{+}$be a continuous unbounded injection, for example, $\alpha: s \mapsto$ $s /(1-s)$. Define

$$
\mu:= \begin{cases}\frac{1}{2 \sup _{t \in \mathbb{R}_{+}} \varphi(t)}, & \text { if } \varphi \text { is bounded } \\ 0, & \text { otherwise. }\end{cases}
$$

If $\mu>0$, let $\operatorname{sat}_{\mu}: \mathbb{R}^{m} \rightarrow \mathcal{B}:=\left\{v \in \mathbb{R}^{m} \mid\|v\| \leq 1\right\}$ be any continuous function with the property that $\operatorname{sat}_{\mu}(e)=\|e\|^{-1} e$ for all $\|e\|>\mu$, in which case the control strategy takes the form

$$
u(t)=-\nu(k(t)) \operatorname{sat}_{\mu}(y(t)-r(t)), \quad k(t)=\alpha(\varphi(t)\|y(t)-r(t)\|) .
$$

In the case $\mu=0$, the control strategy is given formally by

$$
u(t)=-\nu(k(t))\|y(t)-r(t)\|^{-1}(y(t)-r(t)), \quad k(t)=\alpha(\varphi(t)\|y(t)-r(t)\|) .
$$

We accommodate each case and the (potential) discontinuity in (4.2) by embedding the control in a set-valued map $\theta_{\mu}$, defined as follows:

$$
\theta_{\mu}(e)= \begin{cases}\left\{e\|e\|^{-1}\right\}, & \text { if } \quad\|e\|>\mu \\ \mathcal{B}, & \text { if } \quad\|e\| \leq \mu\end{cases}
$$


and interpret both control strategies in the following unified, set-valued sense:

$$
u(t) \in-\nu(k(t)) \theta_{\mu}(y(t)-r(t)), \quad k(t)=\alpha(\varphi(t)\|y(t)-r(t)\|) .
$$

The role of the function $\nu$ is similar to that of a "Nussbaum" [11] function, commonly invoked in adaptive control, see, for example, [4]. If, for a given linear system $(A, B, C)$ of prototype class $\mathcal{L}$, the polarity of the sign-definite high-frequency gain $C B$ is known a priori, then the term $\nu(k(t))$ in $(4.3)$ can be replaced by $k(t)$ if $C B$ is positive definite or by $-k(t)$ if $-C B$ is positive definite.

Care must be exercised in making sense of the closed-loop initial-value problem given by (1.1) and (4.3). The central issue is to establish that $\varphi(t)\|y(t)-r(t)\| \in \operatorname{dom}(\alpha)=[0,1)$ for all $t \in \mathbb{R}_{+}$. This we proceed to demonstrate.

\subsection{Closed-loop system}

Let $\lambda \geq 0, \varphi \in \Phi_{\lambda}, r \in \mathcal{R}$ and let $\mathcal{D} \subset \mathbb{R}_{+} \times \mathbb{R}^{m}$ denote the set

$$
\left\{(t, \xi) \in \mathbb{R}_{+} \times \mathbb{R}^{m} \mid \varphi(t)\|\xi-r(t)\|<1\right\} .
$$

Let $(f, d, T) \in \mathcal{S}$. The conjunction of (1.1) with (4.3) yields the following closed-loop initial-value problem

$$
\dot{y}(t) \in F(t, y(t),(T y)(t)),\left.\quad y\right|_{[-h, 0]}=y^{0} \in C\left([-h, 0], \mathbb{R}^{m}\right),
$$

where the set-valued map $(t, y, w) \mapsto F(t, y, w) \subset \mathbb{R}^{m}$, given by

$$
F(t, y, w):=\left\{f(d(t), w, u) \mid u \in-\nu(\alpha(\varphi(t)\|y-r(t)\|)) \theta_{\mu}(y-r(t))\right\},
$$

is upper semicontinuous on $\mathcal{D} \times \mathbb{R}^{q}$ with non-empty, convex, compact values. By a solution of (4.4) we mean a function $y \in C\left(I, \mathbb{R}^{m}\right)$ on some interval $I$ of the form $[-h, \rho], 0<\rho<\infty$ or $[-h, \omega), 0<\omega \leq \infty$, such that $\left.y\right|_{[-h, 0]}=y^{0},\left.y\right|_{J}$ is locally absolutely continuous, with $(t, y(t)) \in \mathcal{D}$ for all $t \in J$ and $\dot{y}(t) \in F(t, y(t),(T y)(t))$ for almost all $t \in J$, where $J:=I \backslash[-h, 0)$. A solution is said to be maximal if it has no proper right extension that is also a solution. A solution defined on $[-h, \infty)$ is said to be global. We will demonstrate that the control objectives are achieved by establishing that: (i) the initial-value problem (4.4) has a solution; (ii) every solution can be extended to a maximal solution; (iii) every maximal solution is global. Facts (i) and (ii) are a consequence (Cor. 5.2) of the existence theory (Thm. 5.1) developed in Section 5 below; fact (iii) is the essence of the main result in Theorem 6.1. Before proceeding to establish these facts, some commentary on the case $\lambda=0$ is warranted.

\subsubsection{Commentary on the asymptotic tracking problem}

Assume that $\lambda=0$, in which case we have $\mu=0$, and so the formal control structure (4.2) is potentially discontinuous. However, this need not always be the case. For example, with

$$
\nu: k \mapsto k \cos (a k) \quad \text { and } \quad \alpha: s \mapsto \frac{s}{1-s},
$$

where $a>0$, the feedback (4.2) is, in fact, continuous on the domain $\mathcal{D}$ : in particular, the control takes the form

with $\psi \in C\left(\mathcal{D}, \mathbb{R}^{m}\right)$ given by

$$
u(t)=\psi(t, y(t)-r(t))
$$

$$
\psi(t, \xi):=-\cos \left(\frac{a \varphi(t)\|\xi\|}{1-\varphi(t)\|\xi\|}\right)\left(\frac{\varphi(t) \xi}{1-\varphi(t)\|\xi\|}\right) \quad \forall(t, \xi) \in \mathcal{D},
$$

in which case the map $F$ in (4.4) is singleton valued. 


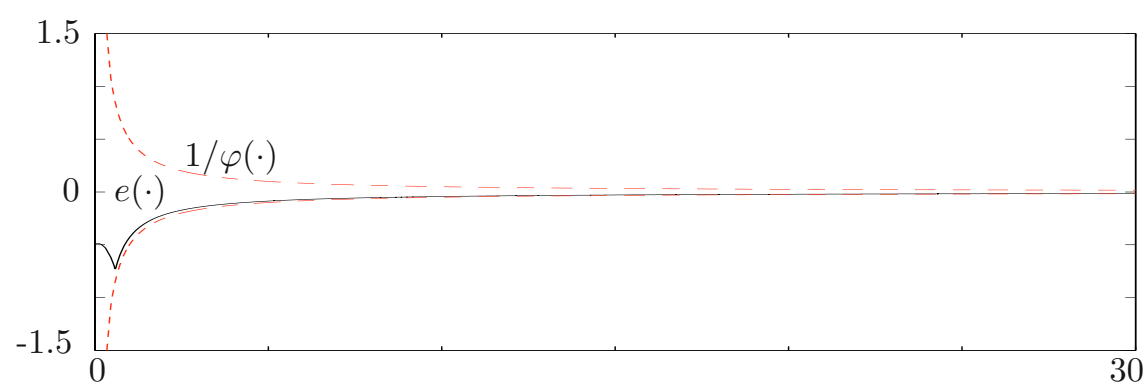

Figure 3. The funnel and tracking error $e$.

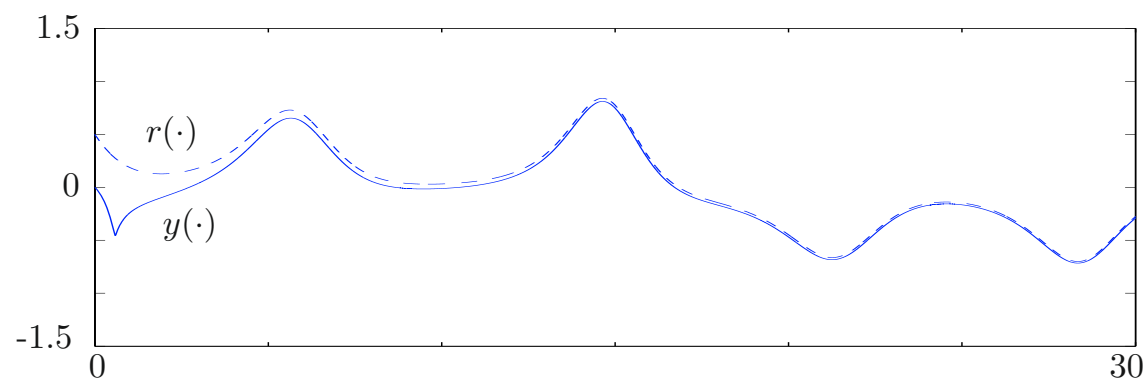

Figure 4. The reference signal $r$ and output $y$.

Example. Consider a single-input, single-output system (3.7) of the nonlinear prototype class, with $f_{1}, f_{2}: \mathbb{R}^{2} \rightarrow$ $\mathbb{R}$ and $g: \mathbb{R} \rightarrow \mathbb{R}$ given by

$$
f_{1}(y, z)=z \sin y, \quad f_{2}(y, z)=-z|z|+y, \quad g(u)=u^{1 / 3}
$$

As reference signal $r \in \mathcal{R}$, we take $r=\zeta_{1} / 2$, where $\zeta_{1}$ is the first component of the (chaotic) solution of the following Lorenz system of equations:

$$
\left.\begin{array}{ll}
\dot{\zeta}_{1}(t)=\zeta_{2}(t)-\zeta_{1}(t), & \zeta_{1}(0)=1, \\
\dot{\zeta}_{2}(t)=c_{0} \zeta_{1}(t)-c_{1} \zeta_{2}(t)-\zeta_{1}(t) \zeta_{3}(t), & \zeta_{2}(0)=0, \\
\dot{\zeta}_{3}(t)=\zeta_{1}(t) \zeta_{2}(t)-c_{2} \zeta_{3}(t), & \zeta_{3}(0)=3,
\end{array}\right\}
$$

with parameter values $c_{0}=28 / 10, c_{1}=1 / 10$ and $c_{2}=8 / 30$. It is well known that the unique global solution of (4.9) is bounded with bounded derivative, see for example [15].

Adopting control parameters $a=1 / 4$ and $\varphi: t \mapsto 2 t$, Figures 3-5 depict the behaviour of the closed-loop system with zero initial state.

There are, of course, practical issues relating to the synthesis of the control strategy (4.6)-(4.7). Whilst later analysis will establish the fact that $\sup _{t \in \mathbb{R}_{+}} \varphi(t)\|y(t)-r(t)\|<1$, and so boundedness of the control function $u$ is assured, practical computation of $u(t)$ for large $t$ may encounter numerical ill-conditioning insofar as it involves the product of "large" and "small" quantities (since $\varphi(t) \rightarrow \infty$ and $\|y(t)-r(t)\| \rightarrow 0$ as $t \rightarrow \infty$ ). These practical issues are not addressed in this paper (the purpose of which is to highlight those performance characteristics that are attainable in principle): however, we remark that the ill-conditioning associated with the case $\mu=0$ may be circumvented (at the expense of some degradation in performance) on setting $\lambda>0$ and replacing unbounded $\varphi$ by a bounded function $\varphi \in \Phi_{\lambda}$ with $\lim \inf _{t \in \mathbb{R}_{+}} \varphi(t)=1 / \lambda$, in which case, the guaranteed performance is weakened to that of approximate tracking, as quantified by $\lim _{\sup } \rightarrow \infty\|y(t)-r(t)\|<\lambda$. 


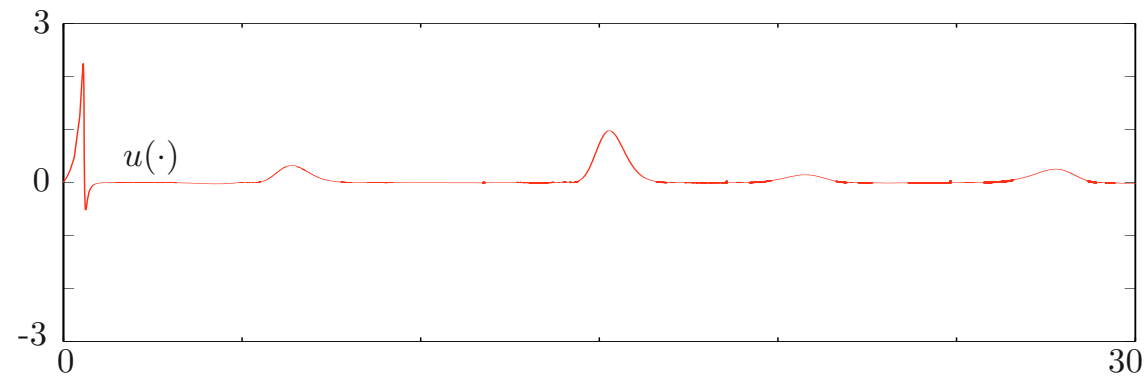

FiguRE 5. The control $u$.

\section{EXISTENCE THEORY}

Here, we present an existence theory of sufficient generality to encompass (4.4). Let $\mathcal{D}$ be a domain in $\mathbb{R}_{+} \times \mathbb{R}^{m}$, that is, a non-empty, connected, relatively open subset of $\mathbb{R}_{+} \times \mathbb{R}^{m}$. Let $(t, y, w) \mapsto G(t, y, w) \subset \mathbb{R}^{m}$ be upper semicontinuous on $\mathcal{G}:=\mathcal{D} \times \mathbb{R}^{q}$, with non-empty, convex and compact values. Let $h \geq 0$ and $T: C\left([-h, \infty), \mathbb{R}^{m}\right) \rightarrow L_{\text {loc }}^{\infty}\left(\mathbb{R}_{+}, \mathbb{R}^{q}\right)$ be a causal operator of class $\mathcal{T}_{h}$. For $t_{0} \geq 0$, consider the initial-value problem

$$
\dot{y}(t) \in G(t, y(t),(T y)(t)),\left.\quad y\right|_{\left[-h, t_{0}\right]}=y^{0} \in C\left(\left[-h, t_{0}\right], \mathbb{R}^{m}\right), \quad\left(t_{0}, y^{0}\left(t_{0}\right)\right) \in \mathcal{D} .
$$

We emphasize that, for reasons which will become apparent in the proof of Theorem 5.1 below, the parameter $t_{0} \geq 0$ has been incorporated in (5.1): this necessitates the obvious generalization of the earlier concept of a solution introduced in the context of (4.4) wherein $t_{0}=0$. Specifically, by a solution of (5.1) we mean a function $y \in C\left(I, \mathbb{R}^{m}\right)$ for some interval $I$ of the form $[-h, \rho], t_{0}<\rho<\infty$ or $[-h, \omega), t_{0}<\omega \leq \infty$, such that $\left.y\right|_{\left[-h, t_{0}\right]}=y^{0},\left.y\right|_{J}$ is locally absolutely continuous, $\dot{y}(t) \in G(t, y(t),(T y)(t))$ for almost all $t \in J$, and $(t, y(t)) \in \mathcal{D}$ for all $t \in J$, where $J:=I \backslash\left[-h, t_{0}\right)$. Again, a solution is said to be maximal if it has no proper right extension that is also a solution.

Theorem 5.1. For each $t_{0} \geq 0$ and $y^{0} \in C\left(\left[-h, t_{0}\right], \mathbb{R}^{m}\right)$ with $\left(t_{0}, y^{0}\left(t_{0}\right)\right) \in \mathcal{D}$,

(i) the initial-value problem (5.1) has a solution;

(ii) every solution can be extended to a maximal solution $y:[-h, \omega) \rightarrow \mathbb{R}^{m}$;

(iii) if $y:[-h, \omega) \rightarrow \mathbb{R}^{m}$ is a maximal solution of (5.1) and $\omega<\infty$, then, for every $\sigma \in\left[t_{0}, \omega\right)$ and every compact set $\mathcal{K} \subset \mathcal{D}$, there exists $t \in[\sigma, \omega)$ such that $(t, y(t)) \notin \mathcal{K}$.

A proof of this result can be found in the Appendix.

Corollary 5.2. Let $(f, d, T) \in \mathcal{S}, \lambda \geq 0$ and $\varphi \in \Phi_{\lambda}$. Then, for every reference signal $r \in \mathcal{R}$ and all initial data $y^{0} \in C\left([-h, 0], \mathbb{R}^{m}\right)$, application of the feedback (4.3) to the system (1.1) yields the initial-value problem (4.4)(4.5) which has a solution and every solution can be extended to a maximal solution $y:[-h, \omega) \rightarrow \mathbb{R}^{m}, 0<\omega \leq$ $\infty$. Furthermore, if $y:[-h, \omega) \rightarrow \mathbb{R}^{m}$ is a maximal solution and there exists a compact set $\mathcal{K} \subset \mathcal{D}$ such that $(t, y(t)) \in \mathcal{K}$ for all $t \in[\sigma, \omega)$, then $\omega=\infty$.

Proof. Defining the domain $\mathcal{D}:=\left\{(t, y) \in \mathbb{R}_{+} \times \mathbb{R}^{m} \mid \varphi(t)\|y-r(t)\|<1\right\}$, we identify the initial-value problem (4.4)-(4.5) as a particular case of (5.1) (with $G=F$ and $t_{0}=0$ ):

$$
\dot{y}(t) \in F(t, y(t),(T y)(t)),\left.\quad y\right|_{[-h, 0]}=y^{0} \in C\left([-h, 0], \mathbb{R}^{m}\right), \quad\left(0, y^{0}(0)\right) \in \mathcal{D},
$$

where $F(t, y, w)=\left\{f(d(t), w, u) \mid u \in-\nu(\alpha(\varphi(t)\|y-r(t)\|)) \theta_{\mu}(y-r(t))\right\}$.

An application of Theorem 5.1 completes the proof. 


\section{MAIN RESULT}

We now arrive at the main result, statement (ii) of which asserts that the output of the closed-loop system evolves within the performance funnel and is bounded away from the funnel boundary.

Theorem 6.1. Let $(f, d, T, h) \in \mathcal{S}, \lambda \geq 0$ and $\varphi \in \Phi_{\lambda}$. Then for every reference signal $r \in \mathcal{R}$ and all initial data $y^{0} \in C\left([-h, 0], \mathbb{R}^{m}\right)$, application of the feedback (4.3) to the system (1.1) yields the closed-loop initial-value problem (4.4)-(4.5) which has a solution and each solution can be extended to a maximal solution $y:[-h, \omega) \rightarrow \mathbb{R}^{m}$. Every maximal solution $y:[-h, \omega) \rightarrow \mathbb{R}^{m}$ has the properties:

(i) $\omega=\infty$;

(ii) $\sup _{t \in \mathbb{R}_{+}} \varphi(t)\|y(t)-r(t)\|<1$;

(iii) the function $k: t \mapsto \alpha(\varphi(t)\|y(t)-r(t)\|)$ is bounded.

Remark 6.2. The conjunction of assertions (i) and (ii) ensures that both control objectives are attained. Assertion (iii) implies boundedness of the control. In the case where $\varphi(t) \rightarrow \infty$ as $t \rightarrow \infty$, assertion (ii) implies asymptotic tracking: $\|y(t)-r(t)\| \rightarrow 0$ as $t \rightarrow \infty$.

Proof. Let $r \in \mathcal{R}$ and $y^{0} \in C\left([-h, 0], \mathbb{R}^{m}\right)$. By Corollary 5.2, the closed-loop initial-value problem (4.4)-(4.5) has a solution and every solution can be maximally extended. Let $y:[-h, \omega) \rightarrow \mathbb{R}^{m}$ be a maximal solution of (4.4). Defining $e(t)=y(t)-r(t)$ for all $t \in[0, \omega)$, we have

$$
\dot{e}(t)+\dot{r}(t) \in F(t, e(t)+r(t),(T y)(t)) \quad \text { for a.a. } t \in[0, \omega) .
$$

Since $(t, y(t)) \in \mathcal{D}$ for all $t \in[0, \omega)$, it follows that $\varphi(t)\|e(t)\|<1$ for all $t \in[0, \omega)$. By properties of $\varphi \in \Phi_{\lambda}$, we may infer boundedness of the function $e$. Furthermore, since $r \in \mathcal{R}$ is bounded, we may conclude that $y$ is bounded. Invoking Assumptions (A3) and (A4) (in particular, property (iv) of the operator class $\mathcal{T}_{h}$ ), we deduce the existence of a non-empty, compact set $\mathcal{K} \subset \mathbb{R}^{p} \times \mathbb{R}^{q}$ such that $(d(t),(T y)(t)) \in \mathcal{K}$ for almost all $t \in[0, \omega)$. With this set, we associate the function $\gamma_{\mathcal{K}}$, defined as in (3.1). Writing

$$
\Sigma:=\{t \in[0, \omega) \mid\|e(t)\|>\mu\}, \quad \text { and } \quad k(t):=\alpha(\varphi(t)\|e(t)\|) \quad \forall t \in[0, \omega),
$$

we have

$$
\begin{aligned}
t \in \Sigma \Longrightarrow\langle e(t) & \left., f\left(d(t),(T y)(t),-\nu(k(t))\|e(t)\|^{-1} e(t)\right)\right\rangle \\
& \leq-\|e(t)\| \min \{\langle u, f(v, w, \nu(k(t)) u)\rangle \mid \quad(v, w) \in \mathcal{K},\|u\|=1\} \\
& =-\|e(t)\| \gamma_{\mathcal{K}}(\nu(k(t))) .
\end{aligned}
$$

Noting that

$$
t \in \Sigma \quad \Longrightarrow \quad F(t, e(t)+r(t),(T y)(t))=\left\{f\left(d(t),(T y)(t),-\nu(k(t))\|e(t)\|^{-1} e(t)\right)\right\},
$$

we may infer from (6.2) that

$$
\langle e(t), v\rangle \leq-\gamma_{\mathcal{K}}(\nu(k(t)))\|e(t)\| \quad \forall v \in F(t, e(t)+r(t),(T y)(t)), \quad \forall t \in \Sigma .
$$

Therefore, by (6.1) and essential boundedness of $\dot{r}$, there exists $c_{0}>0$ such that

$$
\langle e(t), \dot{e}(t)\rangle \leq\left[c_{0}-\gamma_{\mathcal{K}}(\nu(k(t)))\right]\|e(t)\| \quad \text { for a.a. } t \in \Sigma .
$$

By Assumption A2, either (i) $\lim \sup _{s \rightarrow+\infty} \gamma_{\mathcal{K}}(s)=\infty$, or (ii) $\lim \sup _{s \rightarrow-\infty} \gamma_{\mathcal{K}}(s)=\infty$. Therefore, there exists an unbounded sequence $\left(s_{n}\right) \subset \mathbb{R}$, which is either strictly increasing (in case (i)) or strictly decreasing (in case (ii)), such that the sequence $\left(\gamma_{\mathcal{K}}\left(s_{n}\right)\right)$ is unbounded and strictly increasing, with $\gamma_{\mathcal{K}}\left(s_{n}\right)>0$ for 
all $n \in \mathbb{N}$. By properties (4.1) and continuity of $\nu$, for every $a, b \in \mathbb{R}$ the set $\{\kappa>a \mid \nu(\kappa)=b\}$ is non-empty. Let $k_{1} \in\left\{\kappa>\alpha\left(\frac{1}{2}\right) \mid \nu(\kappa)=s_{1}\right\}$ be arbitrary and define the strictly-increasing unbounded sequence $\left(k_{n}\right)$ in $\left(\alpha\left(\frac{1}{2}\right), \infty\right)$ by the recursion $k_{n+1}:=\inf \left\{\kappa>k_{n} \mid \nu(\kappa)=s_{n+1}\right\}$, and so $\gamma_{\mathcal{K}}\left(\nu\left(k_{n}\right)\right)=\gamma_{\mathcal{K}}\left(s_{n}\right) \rightarrow \infty$ as $n \rightarrow \infty$.

We proceed to prove boundedness of $k$. Seeking a contradiction, suppose $k$ is unbounded (in which case, $\operatorname{im}(k)=\operatorname{im}(\alpha)=[\alpha(0), \infty))$. For each $n \in \mathbb{N}$, define

$$
\tau_{n}:=\inf \left\{t \in[0, \omega) \mid k(t)=k_{n+1}\right\} \quad \text { and } \quad \sigma_{n}:=\sup \left\{t \in\left[0, \tau_{n}\right] \mid \gamma_{\mathcal{K}}(\nu(k(t)))=\gamma_{\mathcal{K}}\left(\nu\left(k_{n}\right)\right)\right\} .
$$

We briefly digress to assemble some useful facts.

Proposition 6.3. (a) $\sigma_{n}<\tau_{n} \forall n \in \mathbb{N} . \quad$ (b) $k\left(\sigma_{n}\right)<k\left(\tau_{n}\right) \forall n \in \mathbb{N} . \quad$ (c) $k(t) \geq k_{n} \forall t \in\left[\sigma_{n}, \tau_{n}\right] \forall n \in \mathbb{N}$.
(d) $\gamma_{\mathcal{K}}(\nu(k(t))) \geq \gamma_{\mathcal{K}}\left(\nu\left(k_{n}\right)\right)>0 \quad \forall t \in\left[\sigma_{n}, \tau_{n}\right] \quad \forall n \in \mathbb{N}$.
(e) $\left[\sigma_{n}, \tau_{n}\right] \subset \Sigma \forall n \in \mathbb{N}$.

Proof. (a) Suppose, for contradiction, that $\sigma_{n}=\tau_{n}$ for some $n \in \mathbb{N}$. Then,

$$
\gamma_{\mathcal{K}}\left(s_{n+1}\right)=\gamma_{\mathcal{K}}\left(\nu\left(k_{n+1}\right)\right)=\gamma_{\mathcal{K}}\left(\nu\left(k\left(\tau_{n}\right)\right)\right)=\gamma_{\mathcal{K}}\left(\nu\left(k\left(\sigma_{n}\right)\right)\right)=\gamma_{\mathcal{K}}\left(\nu\left(k_{n}\right)\right)=\gamma_{\mathcal{K}}\left(s_{n}\right),
$$

which contradicts strict monotonicity of the sequence $\left(\gamma_{\mathcal{K}}\left(s_{n}\right)\right)$.

(b) Suppose, for contradiction, that $k\left(\sigma_{n}\right) \geq k\left(\tau_{n}\right)=k_{n+1}$ for some $n \in \mathbb{N}$. Then, since $k(0)=\alpha(0)<$ $\alpha(1 / 2)<k_{n+1}$, there exists $s \leq \sigma_{n}<\tau_{n}$ such that $k(s)=k_{n+1}$, whence the contradiction: $\tau_{n}=\inf \{t \in$ $\left.[0, \omega) \mid k(t)=k_{n+1}\right\} \leq s<\tau_{n}$.

(c) Suppose, for contradiction, that, for some $n \in \mathbb{N}$ and $t \in\left[\sigma_{n}, \tau_{n}\right], k(t)<k_{n}$. Then, since $k\left(\tau_{n}\right)=k_{n+1}$, there exists $s \in\left(\sigma_{n}, \tau_{n}\right]$ such that $k(s)=k_{n}$. Invoking the definition of $\sigma_{n}$, we arrive at a contradiction: $\sigma_{n}<s \leq \sigma_{n}$.

(d) Suppose, for contradiction, that, for some $n \in \mathbb{N}$ and $t \in\left[\sigma_{n}, \tau_{n}\right], \gamma_{\mathcal{K}}(\nu(k(t)))<\gamma_{\mathcal{K}}\left(\nu\left(k_{n}\right)\right)$. Since

$$
\gamma_{\mathcal{K}}\left(\nu\left(k_{n}\right)\right)=\gamma_{\mathcal{K}}\left(s_{n}\right)<\gamma_{\mathcal{K}}\left(s_{n+1}\right)=\gamma_{\mathcal{K}}\left(\nu\left(k_{n+1}\right)\right)=\gamma_{\mathcal{K}}\left(\nu\left(k\left(\tau_{n}\right)\right)\right),
$$

it follows that, for some $s \in\left(\sigma_{n}, \tau_{n}\right], \gamma_{\mathcal{K}}(\nu(k(s)))=\gamma_{\mathcal{K}}\left(\nu\left(k_{n}\right)\right)$, which contradicts the definition of $\sigma_{n}$.

(e) Suppose, for contradiction, that, for some $n \in \mathbb{N}$, there exists $t \in\left[\sigma_{n}, \tau_{n}\right]$ such that $t \notin \Sigma$, then $\|e(t)\| \leq \mu$. Note that $\alpha(0)<\alpha(1 / 2)$ and, if $\mu>0$, then $\alpha(\mu \varphi(t)) \leq \alpha(1 / 2)$. Therefore, we arrive at a contradiction.

$$
\alpha(1 / 2)<k_{n} \leq k(t)=\alpha(\varphi(t)\|e(t)\|) \leq \alpha(1 / 2) .
$$

We now return to the proof of Theorem 6.1. From assertions (c) and (d) of Proposition 6.3, we may infer that

$$
\frac{1}{2}<\alpha^{-1}\left(k_{n}\right) \leq \alpha^{-1}(k(t))=\varphi(t)\|e(t)\|<1 \quad \forall t \in\left[\sigma_{n}, \tau_{n}\right] \quad \forall n \in \mathbb{N},
$$

where $\alpha^{-1}:[\alpha(0), \infty) \rightarrow[0,1)$ is the inverse of the bijection $\alpha:[0,1) \rightarrow \operatorname{im}(\alpha)$, and

$$
-2 \varphi^{2}(t)\|e(t)\| \gamma_{\mathcal{K}}(\nu(k(t))) \leq-\varphi(t) \gamma_{\mathcal{K}}(\nu(k(t))) \quad \forall t \in\left[\sigma_{n}, \tau_{n}\right] \quad \forall n \in \mathbb{N} .
$$

By properties of $\varphi \in \Phi_{\lambda}$, there exists $c_{1}>0$ such that $\dot{\varphi}(t) \leq c_{1}[1+\varphi(t)]$ for almost all $t$ which, together with (6.3), yields, for almost all $t \in \Sigma$,

$$
\begin{aligned}
\frac{\mathrm{d}}{\mathrm{d} t}[\varphi(t)\|e(t)\|]^{2} & =2 \varphi(t) \dot{\varphi}(t)\|e(t)\|^{2}+2 \varphi^{2}(t)\langle e(t), \dot{e}(t)\rangle \\
& \leq 2 c_{1} \varphi(t)[1+\varphi(t)]\|e(t)\|^{2}+2 \varphi^{2}(t)\|e(t)\|\left(c_{0}-\gamma_{\mathcal{K}}(\nu(k(t)))\right) .
\end{aligned}
$$

Invoking (6.4), (6.5) and boundedness of $e$, we may conclude the existence of $c_{2}>0$ such that

$$
\frac{\mathrm{d}}{\mathrm{d} t}[\varphi(t)\|e(t)\|]^{2} \leq \varphi(t)\left[c_{2}-\gamma_{\mathcal{K}}(\nu(k(t)))\right] \quad \text { for a.a. } t \in\left[\sigma_{n}, \tau_{n}\right], \quad \forall n \in \mathbb{N} .
$$


Fix $n \in \mathbb{N}$ sufficiently large so that $c_{2}-\gamma_{\mathcal{K}}\left(\nu\left(k_{n}\right)\right)<0$. Recalling that $\gamma_{\mathcal{K}}(\nu(k(t))) \geq \gamma_{\mathcal{K}}\left(\nu\left(k_{n}\right)\right)$ for all $t \in\left[\sigma_{n}, \tau_{n}\right]$, we have

$$
\frac{\mathrm{d}}{\mathrm{d} t}[\varphi(t)\|e(t)\|]^{2}<0 \quad \text { for a.a. } t \in\left[\sigma_{n}, \tau_{n}\right]
$$

and so $\varphi\left(\tau_{n}\right)\left\|e\left(\tau_{n}\right)\right\|<\varphi\left(\sigma_{n}\right)\left\|e\left(\sigma_{n}\right)\right\|$. Therefore,

$$
k\left(\tau_{n}\right)=\alpha\left(\varphi\left(\tau_{n}\right)\left\|e\left(\tau_{n}\right)\right\|\right)<\alpha\left(\varphi\left(\sigma_{n}\right)\left\|e\left(\sigma_{n}\right)\right\|\right)=k\left(\sigma_{n}\right),
$$

which contradicts assertion (b) of Proposition 6.3. This proves boundedness of $k$ (and so $\nu \circ k: t \mapsto \nu(\alpha(\varphi(t) \| y(t)-$ $r(t) \|))$ is also bounded). By boundedness of $t \mapsto k(t)=\alpha(\varphi(t)\|e(t)\|)$, it follows that $\sup _{t \in[0, \omega)} \varphi(t) \| y(t)-$ $r(t) \|<1$, equivalently, there exists $\varepsilon \in(0,1)$ such that $\varphi(t)\|y(t)-r(t)\| \leq 1-\varepsilon$ for all $t \in[0, \omega)$.

Finally, we show that $\omega=\infty$. By boundedness of $y$, there exists $c_{3}>0$ such that $\|y(t)\| \leq c_{3}$ for all $t \in[0, \omega)$. Suppose $\omega<\infty$. Then

$$
\tilde{\mathcal{K}}:=\left\{(t, v) \in \mathbb{R}_{+} \times \mathbb{R}^{m} \mid \varphi(t)\|v-r(t)\| \leq 1-\varepsilon,\|v\| \leq c_{3}, t \in[0, \omega]\right\}
$$

is a compact subset of $\mathcal{D}$ with the property $(t, y(t)) \in \tilde{\mathcal{K}}$ for all $t \in[0, \omega)$, which contradicts assertion (iii) of Theorem 5.1. Therefore, $\omega=\infty$. This completes the proof.

Remark 6.4. To paraphrase Wonham [17], p. 210, the internal model principle states that every "good" regulator must incorporate a model of the outside world (in the sense that the feedback loop incorporates a suitably reduplicated model of the dynamic structure of the exogenous signals which the closed-loop system is required to track). In the context of linear systems with linear regulators (see $[16,17]$ ), "good" means "structurally stable"; in a more general context of smooth nonlinear systems (see [14]), "good" amounts to a "signal detection" property. In effect, "good" implies some robustness property of the closed loop. The feedback structure proposed in the present paper ensures tracking of any signal of class $W^{1, \infty}\left(\mathbb{R}_{+}, \mathbb{R}^{m}\right)$, yet it does not contain a model capable of replicating this class of signals. For consistency with the internal model principle, one must therefore conclude that the closed-loop system of the present paper lacks certain robustness properties. This perceived lack of robustness may stem from the potential singularity introduced via the injection $\alpha$ in the closed loop or from the unbounded nature of the funnel function $\varphi$. It is not unreasonable to expect that the adoption of a bounded function $\varphi$ (with attendant reduction in performance from asymptotic to approximate tracking) might induce some robustness in the closed loop. However, in the absence of a rigorous robustness analysis, the results of the paper are mainly of a theoretical nature, serving to illustrate those performance characteristics that are attainable, in principle, under weak assumptions on the plant data.

\section{A. Appendix: proof of Theorem 5.1}

Let $X$ be a normed vector space. The open ball of radius $\varepsilon>0$ centred at $x \in X$ is denoted by $\mathbb{B}_{\varepsilon}(x)$ (the ambient space $X$ being clear from context), $\overline{\mathbb{B}}_{\varepsilon}(x)$ denotes the closure of $\mathbb{B}_{\varepsilon}(x)$ : if $x=0$, then, for simplicity, we write $\mathbb{B}_{\varepsilon}$ in place of $\mathbb{B}_{\varepsilon}(0)$.

We record the following properties of $G$ :

(a) $\operatorname{graph}(G):=\{(z, \zeta) \mid \zeta \in G(z), z \in \mathcal{G}\}$ is closed;

(b) if $\mathcal{K} \subset \mathcal{G}$ is compact, then $G(\mathcal{K}):=\cup_{z \in \mathcal{K}} G(z)$ is compact;

$\left.\begin{array}{l}\text { (c) for each } \varepsilon>0 \text {, there exists a locally Lipschitz function } g: \mathcal{G} \rightarrow \mathbb{R}^{m} \\ \text { such that graph }(g) \subset \operatorname{graph}(G)+\mathbb{B}_{\varepsilon} \text {. }\end{array}\right\}$

For (a) see [1], Proposition 2, p. 41, for (b) see [1], Proposition 3, p. 42, for (c) see [1], Theorem 1, p. 84.

To facilitate the proof of the general result in Theorem 5.1, we first establish a variant in the restricted context wherein $G$ is a singleton-valued map $G:(t, y, w) \mapsto\{g(t, y, w)\}$ and $g: \mathcal{G} \rightarrow \mathbb{R}^{m}$ is locally Lipschitz. 
Lemma A.1. Let $g: \mathcal{G} \rightarrow \mathbb{R}^{m}$ be a locally Lipschitz function. For $t_{0} \geq 0$ and $y^{0} \in C\left(\left[-h, t_{0}\right], \mathbb{R}^{m}\right)$, the initial-value problem

$$
\dot{y}(t)=g(t, y(t),(T y)(t)),\left.\quad y\right|_{\left[-h, t_{0}\right]}=y^{0} \in C\left(\left[-h, t_{0}\right], \mathbb{R}^{m}\right), \quad\left(t_{0}, y^{0}\left(t_{0}\right)\right) \in \mathcal{D},
$$

has a unique maximal solution, $y:[-h, \omega) \rightarrow \mathbb{R}^{m}$. Furthermore, if $\omega<\infty$, then, for every $\sigma \in\left[t_{0}, \omega\right)$ and every compact set $\mathcal{K} \subset \mathcal{D}$, there exists $t \in[\sigma, \omega)$ such that $(t, y(t)) \notin \mathcal{K}$.

Proof. Step 1: Existence of a unique solution on a small interval.

By property (iii) of $T \in \mathcal{T}_{h}$, there exist $\delta>0, c_{0}>0$ and $\tau>t_{0}$ such that

$$
\|(T y)(t)-(T z)(t)\| \leq c_{0} \max _{s \in\left[t_{0}, \tau\right]}\|y(s)-z(s)\| \text { for a.a. } t \in\left[t_{0}, \tau\right] \text { and all } y, z \in \mathcal{C}\left(y^{0} ; h, t_{0}, \tau, \delta\right) .
$$

Without loss of generality, we may assume that $\delta \in(0,1)$ and $\tau-t_{0}>0$ are sufficiently small so that $\left[t_{0}, \tau\right] \times$ $\overline{\mathbb{B}}_{\delta}\left(y^{0}\left(t_{0}\right)\right) \subset \mathcal{D}$. For each $\rho \in\left(t_{0}, \tau\right]$, define $C_{\rho}:=\mathcal{C}\left(y^{0}, h, t_{0}, \rho, \delta\right)$ which, equipped with the metric

$$
(y, z) \mapsto \beta_{\rho}(y, z):=\sup _{t \in[-h, \rho]}\|y(t)-z(t)\|,
$$

is a complete metric space. Observe that, if $y \in C_{\rho}$, then $(t, y(t)) \in \mathcal{D}$ for all $t \in\left[t_{0}, \rho\right]$. For each $\rho \in\left(t_{0}, \tau\right]$, define the operator $Z_{\rho}$ on $C_{\rho}$ by

$$
\left(Z_{\rho} y\right)(t):= \begin{cases}y^{0}(t), & t \in\left[-h, t_{0}\right], \\ y^{0}\left(t_{0}\right)+\int_{t_{0}}^{t} g(s, y(s),(T y)(s)) \mathrm{d} s, & t \in\left(t_{0}, \rho\right) .\end{cases}
$$

We proceed to show that $Z_{\rho}$ is a contraction. Define $c_{1}:=\max _{s \in\left[-h, t_{0}\right]}\left\|y^{0}(s)\right\|+\delta$. By property (iv) of $T$, there exists $c_{2}>0$ such that

$$
\sup _{t \in[-h, \tau]}\|y(t)\|<c_{1} \quad \Longrightarrow \quad\|(T y)(t)\|<c_{2} \quad \text { for a.a. } t \in\left[t_{0}, \tau\right] .
$$

By the local Lipschitz property of $g$, there exists a constant $c_{3}>0$ such that, for all $t \in\left[t_{0}, \tau\right]$,

$$
\|g(t, y, w)-g(t, z, x)\| \leq c_{3}[\|y-z\|+\|w-x\|] \quad \forall y, z \in \mathbb{B}_{c_{1}}, \quad \forall w, x \in \mathbb{B}_{c_{2}} .
$$

Write

$$
g^{*}:=\max \left\{\|g(t, y, w)\| \mid \quad(t, y, w) \in\left[t_{0}, \tau\right] \times \overline{\mathbb{B}}_{\delta}\left(y^{0}\left(t_{0}\right)\right) \times \overline{\mathbb{B}}_{c_{2}}\right\} .
$$

Fix $\rho^{*} \in\left(t_{0}, \tau\right]$ sufficiently close to $t_{0}$ so that

$$
\left(\rho^{*}-t_{0}\right)\left(g^{*}+\left(c_{0}+1\right) c_{3}\right)<\delta .
$$

Let $\rho \in\left(t_{0}, \rho^{*}\right]$ and $y \in C_{\rho}$. By definition, $\left.\left(Z_{\rho} y\right)\right|_{\left[-h, t_{0}\right]}=y^{0}$ and

$$
\begin{aligned}
\left\|\left(Z_{\rho} y\right)(t)-y^{0}\left(t_{0}\right)\right\| & =\left\|\int_{t_{0}}^{t} g(s, y(s),(T y)(s)) \mathrm{d} s\right\| \\
& \leq \int_{t_{0}}^{\rho}\|g(s, y(s),(T y)(s))\| \mathrm{d} s \leq\left(\rho-t_{0}\right) g^{*}<\delta \quad \forall t \in\left[t_{0}, \rho\right] .
\end{aligned}
$$


Therefore $\left(Z_{\rho} y\right)(\cdot) \in C_{\rho}$. Furthermore,

$$
\begin{aligned}
\beta_{\rho}\left(Z_{\rho} y, Z_{\rho} z\right) & =\sup _{t \in\left[t_{0}, \rho\right]}\left\|\int_{t_{0}}^{t}[g(s, y(s),(T y)(s))-g(s, z(s),(T z)(s))] \mathrm{d} s\right\| \\
& \leq \int_{t_{0}}^{\rho}\|g(s, y(s),(T y)(s))-g(s, z(s),(T z)(s))\| \mathrm{d} s \\
& \left.\leq\left(\rho-t_{0}\right) c_{3}\left[\begin{array}{c}
\operatorname{ess-sup} \\
s \in\left[t_{0}, \rho\right]
\end{array}\right](T y)(s)-(T z)(s) \|+\beta_{\rho}(y, z)\right] \\
& \leq\left(c_{0}+1\right)\left(\rho-t_{0}\right) c_{3} \beta_{\rho}(y, z) \quad \forall y, z \in C_{\rho},
\end{aligned}
$$

wherein the last inequality follows by (A.3). Since $\left(c_{0}+1\right)\left(\rho-t_{0}\right) c_{3}<\delta<1$, we may infer that $Z_{\rho}: C_{\rho} \rightarrow C_{\rho}$ is a contraction. By the contraction mapping theorem, $Z_{\rho}$ has a unique fixed point. Thus we have shown that, for each $\rho \in\left(t_{0}, \rho^{*}\right]$, the initial-value problem (A.2) has a unique solution $y \in C_{\rho}$. We stress that the uniqueness property of $y$ holds only in relation to solutions in the restricted class $C_{\rho}$ : there may exist another solution on the interval $[-h, \rho]$ which is not contained in the space $C_{\rho}$. However, the following argument establishes uniqueness of the solution on a sufficiently small interval. Let $y^{*}$ (not necessarily in $C_{\rho^{*}}$ ) be a solution on $\left[-h, \rho^{*}\right]$. Define

$$
\Delta:=\left\{t \in\left[t_{0}, \rho^{*}\right] \mid\left\|y^{*}(t)-y^{0}\left(t_{0}\right)\right\|=\delta\right\}, \quad \rho:= \begin{cases}\inf \Delta, & \Delta \neq \emptyset \\ \rho^{*}, & \Delta=\emptyset .\end{cases}
$$

Clearly $\rho>t_{0}$ and $y:=\left.y^{*}\right|_{[-h, \rho]}$ is in $C_{\rho}$. Therefore, $y$ is the unique solution of (A.2) on the interval $[-h, \rho]$.

Step 2: Extended uniqueness: any two solutions must coincide on the intersection of their domains.

Let $y_{1}: I_{1} \rightarrow \mathbb{R}^{m}$ and $y_{2}: I_{2} \rightarrow \mathbb{R}^{m}$ be solutions of (A.2) and, without loss of generality, assume $I_{2} \subset I_{1}$. For contradiction, suppose that $\left.y_{1}\right|_{I_{2}} \neq y_{2}$. Let $t^{*}:=\inf \left\{t \in I_{2} \mid y_{1}(t) \neq y_{2}(t)\right\}$. By the result in Step 1 , the solutions $y_{1}$ and $y_{2}$ must coincide on some interval $[-h, \rho]$, with $\rho>t_{0}$. Therefore, $t^{*}>t_{0}$. An application of the result of Step 1 in the context of an initial-value problem of the form (A.2), with $t^{*}$ replacing $t_{0}$ and initial function $\left.y_{1}\right|_{\left[-h, t^{*}\right]} \in C\left(\left[-h, t^{*}\right], \mathbb{R}^{m}\right)$ replacing $y^{0}$, yields the existence of a unique solution $y \in C\left([-h, \rho], \mathbb{R}^{m}\right)$ for some $\rho>t^{*}$. It follows that $y_{1}(t)=y_{2}(t)=y(t)$ for all $t \in[-h, \rho]$, contradicting the definition of $t^{*}$.

Step 3: Existence of a unique maximal solution.

Let $\mathcal{P}$ be the set of all $\rho>t_{0}$ such that there exists a solution $y_{\rho}$ of (A.2) on the interval $[-h, \rho]$. By Step 1, we know that $\mathcal{P} \neq \emptyset$. Let $\omega:=\sup \mathcal{P}$ and define $y:[-h, \omega) \rightarrow \mathbb{R}^{m}$ by the property

$$
\left.y\right|_{[-h, \rho]}=y_{\rho} \quad \forall \rho \in \mathcal{P} .
$$

The function $y$ is well-defined since, by Step 2, for all $\rho_{1}, \rho_{2} \in \mathcal{P}$, we have $y_{\rho_{2}}=\left.y_{\rho_{1}}\right|_{\left[-h, \rho_{2}\right]}$ whenever $\rho_{2} \leq \rho_{1}$. Clearly $y$ is a maximal solution and uniqueness follows by Step 2 .

Step 4: Assume that $y:[-h, \omega) \rightarrow \mathbb{R}^{m}$ is a maximal solution with $\omega<\infty$. Seeking a contradiction, suppose there exist $\sigma \in\left[t_{0}, \omega\right)$ and a compact set $\mathcal{K} \subset \mathcal{D}$ such that $(t, y(t)) \in \mathcal{K}$ for all $t \in[\sigma, \omega)$. Then $y$ is bounded and, by property (iv) of $T \in \mathcal{T}_{h}, T y$ is essentially bounded. Therefore, the function $t \mapsto(t, y(t),(T y)(t))$ is essentially bounded and so, by continuity of $g$, it follows that $\dot{y}$ is essentially bounded on the interval $\left[t_{0}, \omega\right)$. Therefore $y$ is uniformly continuous on $[-h, \omega)$ and so extends to $y^{*} \in C\left([-h, \omega], \mathbb{R}^{m}\right)$. By compactness of $\mathcal{K}$, we have $\left(\omega, y^{*}(\omega)\right) \in \mathcal{K} \subset \mathcal{D}$. An application of the result of Step 1 in the context of an initial-value problem of the form (A.2), with $\omega$ replacing $t_{0}$ and $y^{*}$ replacing $y^{0}$, yields the existence of a unique solution $y^{e} \in C\left([-h, \rho], \mathbb{R}^{m}\right)$ for some $\rho>\omega$, with $\left.y^{e}\right|_{[-h, \omega)}=y$. This contradicts maximality of $y$.

We are now in a position to prove the existence of a solution to the problem (5.1). 


\section{Proof of Theorem 5.1}

Proof. (i) Let $\left(\varepsilon_{n}\right) \subset(0,1)$ be a monotonically decreasing sequence with $\varepsilon_{n} \rightarrow 0$ as $n \rightarrow \infty$. By property (A.1c), for each $n \in \mathbb{N}$, there exists a locally Lipschitz function $g_{n}: \mathcal{G} \rightarrow \mathbb{R}^{m}$ with

$$
\operatorname{graph}\left(g_{n}\right) \subset \operatorname{graph}(G)+\mathbb{B}_{\varepsilon_{n}} .
$$

By Lemma A.1, for each $n \in \mathbb{N}$, the initial-value problem

$$
\dot{y}(t)=g_{n}(t, y(t),(T y)(t)),\left.\quad y\right|_{\left[-h, t_{0}\right]}=y^{0} \in C\left(\left[-h, t_{0}\right], \mathbb{R}^{m}\right), \quad\left(t_{0}, y^{0}\left(t_{0}\right)\right) \in \mathcal{D},
$$

has a unique maximal solution which we denote by $y_{n}:\left[-h, \omega_{n}\right) \rightarrow \mathbb{R}^{m}$.

Recalling that $\mathcal{D}$ is a relatively open subset of $\mathbb{R}_{+} \times \mathbb{R}^{m}$ and invoking property (iii) of $T \in \mathcal{T}_{h}$, we may choose $\delta>0$ sufficiently small and $\omega^{*}>t_{0}$ sufficiently close to $t_{0}$ so that

$$
\left[t_{0}, \omega^{*}\right] \times \overline{\mathbb{B}}_{\delta}\left(y^{0}\left(t_{0}\right)\right)=: \mathcal{K}_{0} \subset \mathcal{D}
$$

and there exists $c_{0}>0$ such that

$$
\operatorname{ess-sup}_{t \in\left[t_{0}, \omega^{*}\right]}\|(T y)(t)-(T z)(t)\| \leq c_{0} \max _{t \in\left[t_{0}, \omega^{*}\right]}\|y(t)-z(t)\| \quad \forall y, z \in \mathcal{C}\left(y^{0} ; h, t_{0}, \omega^{*}, \delta\right) .
$$

For each $n \in \mathbb{N}$, define

$$
\omega_{n}^{*}:=\min \left\{\omega^{*}, \omega_{n}\right\}, \Delta_{n}:=\left\{t \in\left[t_{0}, \omega_{n}^{*}\right) \mid\left\|y_{n}(t)-y^{0}\left(t_{0}\right)\right\|=\delta\right\}, \quad \rho_{n}:= \begin{cases}\inf \Delta_{n}, & \text { if } \Delta_{n} \neq \emptyset \\ \omega_{n}^{*}, & \text { if } \Delta_{n}=\emptyset\end{cases}
$$

We claim that $\rho_{n}<\omega_{n}$ for all $n \in \mathbb{N}$. Suppose otherwise, then there exists $n \in \mathbb{N}$ such that $\rho_{n}=\omega_{n}$. It follows that $\Delta_{n}=\emptyset$ and so $\omega_{n}=\omega_{n}^{*} \leq \omega^{*}$. Therefore, $\left(t, y_{n}(t)\right) \in \mathcal{K}_{0} \subset \mathcal{D}$ for all $t \in\left[t_{0}, \omega_{n}\right)$, contradicting the final assertion of Lemma A.1. Therefore, $\rho_{n}<\omega_{n}$ for all $n \in \mathbb{N}$. Furthermore, for each $n \in \mathbb{N}, y_{n}(t) \in \overline{\mathbb{B}}_{\delta}\left(y^{0}\left(t_{0}\right)\right)$ for all $t \in\left[t_{0}, \rho_{n}\right]$ and so

$$
\left\|y_{n}(t)\right\| \leq c_{1}:=\max _{s \in\left[-h, t_{0}\right]}\left\|y^{0}(s)\right\|+\delta \quad \text { for all } t \in\left[-h, \rho_{n}\right] \text { and all } n \in \mathbb{N} .
$$

By property (iv) of $T \in \mathcal{T}_{h}$, there exists $c_{2}>0$ such that

$$
\left\|\left(T y_{n}\right)(t)\right\| \leq c_{2} \quad \text { for a.a. } t \in\left[t_{0}, \rho_{n}\right] \text { and all } n \in \mathbb{N} .
$$

Write $\mathcal{K}_{1}:=\mathcal{K}_{0} \times \overline{\mathbb{B}}_{c_{2}}$ and observe

$$
\left(t, y_{n}(t),\left(T y_{n}\right)(t)\right) \in \mathcal{K}_{1} \quad \text { for a.a. } t \in\left[t_{0}, \rho_{n}\right] \text { and all } n \in \mathbb{N} .
$$

By property (A.1b) of $G$, the set $\mathcal{K}_{2}:=G\left(\mathcal{K}_{1}\right)$ is compact. Let $c_{3}:=1+\max _{v \in \mathcal{K}_{2}}\|v\|$. Then, in view of (A.4),

$$
\left\|g_{n}(t, y, w)\right\|<c_{3} \quad \text { for all }(t, y, w) \in \mathcal{K}_{1} \text { and all } n \in \mathbb{N} .
$$

Therefore,

$$
\begin{aligned}
\left\|y_{n}\left(\rho_{n}\right)-y^{0}\left(t_{0}\right)\right\| \leq \int_{t_{0}}^{\rho_{n}}\left\|\dot{y}_{n}(t)\right\| \mathrm{d} t & =\int_{t_{0}}^{\rho_{n}}\left\|g_{n}\left(t, y_{n}(t),\left(T y_{n}\right)(t)\right)\right\| \mathrm{d} t \\
& <c_{3}\left|\rho_{n}-t_{0}\right| \quad \forall n \in \mathbb{N} .
\end{aligned}
$$


Next, define $\rho:=\inf _{n \in \mathbb{N}} \rho_{n} \geq t_{0}$. Seeking a contradiction, suppose $\rho=t_{0}$. Fix $n \in \mathbb{N}$ sufficiently large so that $c_{3}\left|\rho_{n}-t_{0}\right|<\delta$ and $\rho_{n}<\omega^{*}$. Recalling that $\rho_{n}<\omega_{n}$, we have $\rho_{n}<\min \left\{\omega^{*}, \omega_{n}\right\}=\omega_{n}^{*}$ and so $\Delta_{n} \neq \emptyset$ and we arrive at a contradiction:

Therefore $\rho \in\left(t_{0}, \omega^{*}\right]$. For each $n \in \mathbb{N}$, define

$$
\delta=\left\|y_{n}\left(\rho_{n}\right)-y^{0}\left(t_{0}\right)\right\|<c_{3}\left|\rho_{n}-t_{0}\right|<\delta .
$$

$$
z_{n}:=\left.y_{n}\right|_{\left[t_{0}, \rho\right]} \quad \text { and } \quad w_{n}:=\left.\left(T y_{n}\right)\right|_{\left[t_{0}, \rho\right]} .
$$

For all $t \in\left[t_{0}, \rho\right],\left(z_{n}(t)\right) \subset \overline{\mathbb{B}}_{\delta}\left(y^{0}\left(t_{0}\right)\right)$ and by (A.6),

$$
\left\|\dot{z}_{n}(t)\right\|<c_{3} \text { for a.a. } t \in\left[t_{0}, \rho\right] \text { and all } n \in \mathbb{N} .
$$

Therefore, the sequence $\left(z_{n}\right) \subset C\left(\left[t_{0}, \rho\right], \mathbb{R}^{m}\right)$ is uniformly bounded and equicontinuous. By the Arzelà-Ascoli theorem, and extracting a subsequence if necessary, we may assume that $\left(z_{n}\right)$ converges uniformly to $z \in$ $C\left(\left[t_{0}, \rho\right], \mathbb{R}^{m}\right)$.

To complete the proof of assertion (i), we adopt an argument akin to that used in the proof of [3], Theorem 3.1.7 and [9], Theorem 2D.5.

By weak*-compactness of the unit ball in $L^{\infty}\left(\left[t_{0}, \rho\right], \mathbb{R}^{m}\right)$ (Alaoglu's theorem), together with (A.8), the sequence $\left(\dot{z}_{n}\right) \subset L^{\infty}\left(\left[t_{0}, \rho\right], \mathbb{R}^{m}\right) \subset L^{1}\left(\left[t_{0}, \rho\right], \mathbb{R}^{m}\right)$ has a subsequence (which we do not relabel) with weak*-limit $v \in L^{\infty}\left(\left[t_{0}, \rho\right], \mathbb{R}^{m}\right)$, that is,

$$
\lim _{n \rightarrow \infty} \int_{t_{0}}^{\rho}\left\langle p(t), \dot{z}_{n}(t)\right\rangle \mathrm{d} t=\int_{t_{0}}^{\rho}\langle p(t), v(t)\rangle \mathrm{d} t \quad \forall p \in L^{1}\left(\left[t_{0}, \rho\right], \mathbb{R}^{m}\right)
$$

and so, a fortiori, the sequence $\left(\dot{z}_{n}\right)$ converges weakly in $L^{1}\left(\left[t_{0}, \rho\right], \mathbb{R}^{m}\right)$ to $v$. Let $\left\{e_{1}, \ldots, e_{m}\right\}$ be a basis for $\mathbb{R}^{m}$. For $k=1, \ldots, m$ and $t \in\left[t_{0}, \rho\right]$, define $p_{k, t} \in L^{1}\left(\left[t_{0}, \rho\right], \mathbb{R}^{m}\right)$ by

$$
p_{k, t}(s):= \begin{cases}e_{k}, & s \in\left[t_{0}, t\right] \\ 0, & \text { otherwise. }\end{cases}
$$

Setting $p=p_{k, t}\left(k=1, \ldots, m\right.$ and $\left.t \in\left[t_{0}, \rho\right]\right)$ in (A.9) and integrating, we may now conclude that

$$
z(t)=\lim _{n \rightarrow \infty} z_{n}(t)=y^{0}\left(t_{0}\right)+\int_{t_{0}}^{t} v(s) \mathrm{d} s \quad \forall t \in\left[t_{0}, \rho\right] .
$$

Therefore, $z \in A C\left(\left[t_{0}, \rho\right], \mathbb{R}^{m}\right)$ (the space of absolutely continuous functions $\left.\left[t_{0}, \rho\right] \rightarrow \mathbb{R}^{m}\right)$ and $\dot{z}(t)=v(t)$ for almost all $t \in\left[t_{0}, \rho\right]$.

Let $y \in C\left([-h, \rho], \mathbb{R}^{m}\right)$ denote the concatenation of $y^{0}$ and $z$, and write $w:=\left.(T y)\right|_{\left[t_{0}, \rho\right]}$. Therefore, $\left.y\right|_{\left[-h, t_{0}\right]}=$ $y^{0},\left.y\right|_{\left[t_{0}, \rho\right]}=z \in A C\left(\left[t_{0}, \rho\right], \mathbb{R}^{m}\right)$ and, to conclude that $y$ is a solution of the initial-value problem (5.1), it suffices to show that $\dot{z}(t) \in G(t, z(t), w(t))$ for almost all $t \in\left[t_{0}, \rho\right]$.

By (A.5), we have

$$
\left\|w_{n}(t)-w(t)\right\| \leq c_{0} \max _{s \in\left[t_{0}, \rho\right]}\left\|z_{n}(s)-z(s)\right\| \quad \text { for a.a. } t \in\left[t_{0}, \rho\right] \text { and all } n \in \mathbb{N} .
$$

Therefore, for almost all $t \in\left[t_{0}, \rho\right], w_{n}(t) \rightarrow w(t)$ as $n \rightarrow \infty$. Moreover,

$$
\int_{t_{0}}^{\rho}\left\|w_{n}(t)-w(t)\right\| \mathrm{d} t \leq c_{0}\left|\rho-t_{0}\right| \max _{s \in\left[t_{0}, \rho\right]}\left\|z_{n}(s)-z(s)\right\| \rightarrow 0 \quad \text { as } n \rightarrow \infty .
$$

Therefore, $\left(w_{n}\right)$ converges (strongly) in $L^{1}\left(\left[t_{0}, \rho\right], \mathbb{R}^{m}\right)$ to $w$. 
Define the function $\sigma: \mathcal{K}_{1} \times \mathbb{R}^{m} \rightarrow \mathbb{R}$ by

$$
\sigma(t, \eta, \xi, q):=\max \{\langle q, \zeta\rangle \mid \zeta \in G(t, \eta, \xi)\}
$$

Observe that, for each $(t, \eta, \xi) \in \mathcal{K}_{1}, q \mapsto \sigma(t, \eta, \xi, q)$ is the support function for the compact and convex set $G(t, \eta, \xi)$ (and so is globally Lipschitz). Therefore, to establish that $\dot{z}(t) \in G(t, z(t), w(t))$ for almost all $t \in\left[t_{0}, \rho\right]$, it suffices to show that

$$
\langle q, \dot{z}(t)\rangle \leq \sigma(t, z(t), w(t), q) \quad \text { for a.a. } t \in\left[t_{0}, \rho\right] \text { and all } q \in \mathbb{R}^{m} \text {. }
$$

By continuity of the maps $q \mapsto\langle q, \zeta\rangle$ and $q \mapsto \sigma(t, \eta, \xi, q)$ for all $\zeta \in \mathbb{R}^{m}$ and all $(t, \eta, \xi) \in \mathcal{K}_{1}$, (A.11) holds if, any only if,

$$
\langle q, \dot{z}(t)\rangle \leq \sigma(t, z(t), w(t), q) \quad \text { for a.a. } t \in\left[t_{0}, \rho\right] \text { and all } q \in \mathbb{Q}^{m},
$$

where $\mathbb{Q}^{m} \subset \mathbb{R}^{m}$ is the set of vectors in $\mathbb{R}^{m}$ with rational coordinates. We proceed to establish (A.12). First, we show that, for each $q \in \mathbb{R}^{m}$, the map $(t, \eta, \xi) \mapsto \sigma(t, \eta, \xi, q)$ is upper semicontinuous on $\mathcal{G}$. Let $q \in \mathbb{R}^{m}$ and $(t, \eta, \xi) \in \mathcal{K}_{1}$ be arbitrary and define

$$
\sigma^{*}:=\limsup _{\left(t^{\prime}, \eta^{\prime}, \xi^{\prime}\right) \rightarrow(t, \eta, \xi)} \sigma\left(t^{\prime}, \eta^{\prime}, \xi^{\prime}, q\right)
$$

Let $\left(\left(t_{k}, \eta_{k}, \xi_{k}\right)\right) \subset \mathcal{K}_{1}$ be a sequence converging to $(t, \eta, \xi)$ such that $\sigma\left(t_{k}, \eta_{k}, \xi_{k}, q\right) \rightarrow \sigma^{*}$ as $k \rightarrow \infty$. For each $k \in \mathbb{N}$, by compactness of $G\left(t_{k}, \eta_{k}, \xi_{k}\right)$ there exists $\zeta_{k} \in G\left(t_{k}, \eta_{k}, \xi_{k}\right)$ such that $\left\langle q, \zeta_{k}\right\rangle=\sigma\left(t_{k}, \eta_{k}, \xi_{k}, q\right)$. The resulting sequence $\left(\zeta_{k}\right)$ is contained in the compact set $\mathcal{K}_{2}=G\left(\mathcal{K}_{1}\right)$ and so has a subsequence converging to $\zeta \in \mathcal{K}_{2}$. By property (A.1a), the graph of $G$ is closed and so we may infer that $\zeta \in G(t, \eta, \xi)$. Therefore,

$$
\limsup _{\left(t^{\prime}, \eta^{\prime}, \xi^{\prime}\right) \rightarrow(t, \eta, \xi)} \sigma\left(t^{\prime}, \eta^{\prime}, \xi^{\prime}, q\right)=\lim _{k \rightarrow \infty} \sigma\left(t_{k}, \eta_{k}, \xi_{k}, q\right)=\lim _{k \rightarrow \infty}\left\langle q, \zeta_{k}\right\rangle=\langle q, \zeta\rangle \leq \sigma(t, \eta, \xi, q),
$$

whence upper semicontinuity of $\sigma(\cdot, \cdot, \cdot, q)$.

For $p \in L^{\infty}\left(\left[t_{0}, \rho\right], \mathbb{R}^{m}\right)$,

$$
\left|\sigma\left(t, z_{n}(t), w_{n}(t), p(t)\right)\right| \leq \max _{v \in \mathcal{K}_{2}}\|v\|\|p(t)\| \leq c_{3}\|p(t)\| \quad \text { for a.a. } t \in\left[t_{0}, \rho\right] \text { and all } n \in \mathbb{N} .
$$

Furthermore, in view of (A.4),

$$
\begin{aligned}
\left\langle p(t), \dot{z}_{n}(t)\right\rangle & =\left\langle p(t), g_{n}\left(t, z_{n}(t), w_{n}(t)\right)\right\rangle \\
& \leq \sigma\left(t, z_{n}(t), w_{n}(t), p(t)\right)+\varepsilon_{n}\|p(t)\| \quad \text { for a.a. } t \in\left[t_{0}, \rho\right] \text { and all } n \in \mathbb{N},
\end{aligned}
$$

and so

$$
\int_{t_{0}}^{\rho}\left[\left\langle p(t), \dot{z}_{n}(t)\right\rangle-\varepsilon_{n}\|p(t)\|\right] \mathrm{d} t \leq \int_{t_{0}}^{\rho} \sigma\left(t, z_{n}(t), w_{n}(t), p(t)\right) \mathrm{d} t \quad \forall n \in \mathbb{N} .
$$

Taking the limit superior as $n \rightarrow \infty$, invoking Fatou's lemma and upper semicontinuity of $\sigma(\cdot, \cdot, \cdot, q)$, we have

$$
\int_{t_{0}}^{\rho}\langle p(t), \dot{z}(t)\rangle \mathrm{d} t \leq \int_{t_{0}}^{\rho} \sigma(t, z(t), w(t), p(t)) \mathrm{d} t .
$$

Let $q \in \mathbb{Q}^{m}$ and let $t \in\left[t_{0}, \rho\right)$ be a Lebesgue point for the integrable functions $\dot{z}$ and $t \mapsto \sigma(t, z(t), w(t), q)$. For $\tau>0$, define $p \in L^{\infty}\left(\left[t_{0}, \rho\right], \mathbb{R}^{m}\right)$ by

$$
p(s):= \begin{cases}q / \tau, & s \in[t, t+\tau] \cap\left[t_{0}, \rho\right], \\ 0, & \text { otherwise. }\end{cases}
$$


By (A.13), we have

$$
\frac{1}{\tau} \int_{t}^{t+\tau}[\sigma(s, z(s), w(s), q)-\langle q, \dot{z}(s)\rangle] \mathrm{d} s \geq 0 \quad \forall \tau>0 .
$$

Passage to the limit as $\tau \rightarrow 0$ yields $\langle q, \dot{z}(t)\rangle \leq \sigma(t, z(t), w(t), q)$, which is valid for all $t \in\left[t_{0}, \rho\right] \backslash \mathcal{N}(q)$, where $\mathcal{N}(q)$ is a set of measure zero which may depend on $q \in \mathbb{Q}^{m}$. Since $\mathbb{Q}^{m}$ is countable, $\cup_{q \in \mathbb{Q}^{m}} \mathcal{N}(q)$ has measure zero and so we may conclude that (A.12) (and hence (A.11)) holds. We have now shown that $y:[-h, \rho] \rightarrow \mathbb{R}^{m}$ is a solution of (5.1), whence assertion (i).

(ii) Let $y \in C\left(I_{y}, \mathbb{R}^{m}\right)$ be a solution of (5.1). Define

$$
\mathcal{A}:=\left\{(I, z) \mid I_{y} \subset I, z \in C\left(I, \mathbb{R}^{m}\right) \text { is a solution of (5.1) with }\left.z\right|_{I_{y}}=y\right\} .
$$

On this non-empty set define a partial order $\preceq$ by

$$
\left(I_{1}, z_{1}\right) \preceq\left(I_{2}, z_{2}\right) \quad \Longleftrightarrow \quad \sup I_{1} \leq \sup I_{2} \quad \text { and }\left.\quad z_{2}\right|_{I_{1}}=z_{1} .
$$

We proceed to show that $\mathcal{A}$ has a maximal element, that is, an element $\left(I^{*}, z^{*}\right) \in \mathcal{A}$ such that, for all $(I, z) \in \mathcal{A}$, $\left(I^{*}, z^{*}\right) \preceq(I, z)$ implies $(I, z)=\left(I^{*}, z^{*}\right)$, in which case $z^{*} \in C\left(I^{*}, \mathbb{R}^{m}\right)$ is a solution of $(5.1)$ and is a maximal extension of the solution $y \in C\left(I_{y}, \mathbb{R}^{m}\right)$. Let $\mathcal{O}$ be a totally ordered subset of $\mathcal{A}$. Let $\omega:=\sup \{\sup I \mid(I, z) \in \mathcal{O}\}$ and let $z^{*}:[-h, \omega) \rightarrow \mathbb{R}^{m}$ be defined by the property that, for every $(I, z) \in \mathcal{O},\left.z^{*}\right|_{I}=z$. Then $\left(\omega, z^{*}\right)$ is in $\mathcal{A}$ and is an upper bound for $\mathcal{O}$. By Zorn's Lemma, it follows that $\mathcal{A}$ contains at least one maximal element. This establishes assertion (ii).

(iii) Assume $y \in C\left([-h, \omega), \mathbb{R}^{m}\right)$ is a maximal solution of (5.1) and that $\omega<\infty$. Seeking a contradiction, suppose there exist $\sigma \in\left[t_{0}, \omega\right)$ and compact $\mathcal{K} \subset \mathcal{D}$ such that $(t, y(t)) \in \mathcal{K}$ for all $t \in[\sigma, \omega)$. By boundedness of $y$ and property (iv) of $\mathcal{T}_{h}$, we conclude that $T y$ is bounded. Therefore, the function $t \mapsto(t, y(t),(T y)(t))$ is essentially bounded and so by property (A.1b) of $G$, it follows that $\dot{y}$ is essentially bounded on $[\sigma, \omega)$. Therefore, $y$ is uniformly continuous on $[-h, \omega)$ and so extends to a function $y^{*} \in C\left([-h, \omega], \mathbb{R}^{m}\right)$. By compactness of $\mathcal{K}$, we have $\left(\omega, y^{*}(\omega)\right) \in \mathcal{K} \subset \mathcal{D}$. An application of Assertion (i) of the theorem (with $\omega$ and $y^{*}$ replacing $t_{0}$ and $y^{0}$, respectively) yields the existence of a solution $y^{e} \in C\left([-h, \rho], \mathbb{R}^{m}\right)$ for some $\rho>\omega$, with $\left.y^{e}\right|_{[-h, \omega)}=y$. This contradicts maximality of $y$.

\section{REFERENCES}

[1] J.P. Aubin and A. Cellina, Differential Inclusions. Springer-Verlag, Berlin (1984).

[2] M. Brokate and J. Sprekels, Hysteresis and Phase Transitions. Springer, New York (1996).

[3] F.H. Clarke, Optimization and Nonsmooth Analysis. Wiley-Interscience, New York (1983).

[4] A. Ilchmann, Non-Identifier-Based High-Gain Adaptive Control. Springer-Verlag, London (1993).

[5] A. Ilchmann, E.P. Ryan and C.J. Sangwin, Systems of controlled functional differential equations and adaptive tracking. SIAM J. Contr. Opt. 40 (2002) 1746-1764.

[6] A. Ilchmann, E.P. Ryan and C.J. Sangwin, Tracking with prescribed transient behaviour. ESAIM: COCV 7 (2002) $471-493$.

[7] A. Ilchmann, E.P. Ryan and P.N. Townsend, Tracking control with prescribed transient behaviour for systems of known relative degree. Systems Control Lett. 55 (2006) 396-406.

[8] A. Ilchmann, E.P. Ryan and P.N. Townsend, Tracking with prescribed transient behaviour for nonlinear systems of known relative degree. SIAM J. Contr. Opt. 46 (2007) 210-230.

[9] P.D. Loewen, Optimal Control via Nonsmooth Analysis, CRM Proc. \& Lecture Notes 2. AMS, Providence RI (1993).

[10] H. Logemann and A.D. Mawby, Low-gain integral control of infinite dimensional regular linear systems subject to input hysteresis, in Advances in Mathematical Systems Theory, F. Colonius, U. Helmke, D. Prätzel-Wolters and F. Wirth Eds., Birkhäuser Verlag, Boston (2001) 255-293.

[11] R.D. Nussbaum, Some remarks on a conjecture in parameter adaptive control. Systems Control Lett. 3 (1983) $243-246$.

[12] E.P. Ryan and C.J. Sangwin, Controlled functional differential equations and adaptive stabilization. Int. J. Control 74 (2001) $77-90$.

[13] E.D. Sontag, Smooth stabilization implies coprime factorization. IEEE Trans. Autom. Control 34 (1989) 435-443.

[14] E.D. Sontag, Adaptation and regulation with signal detection implies internal model. Systems Control Lett. 50 (2003) 119-126.

[15] C. Sparrow, The Lorenz Equations: Bifurcations, Chaos and Strange Attractors. Springer-Verlag, New York (1982).

[16] M. Vidyasagar, Control System Synthesis: A Factorization Approach. MIT Press, Cambridge (1985).

[17] W.M. Wonham, Linear Multivariable Control: A Geometric Approach. 2nd edn., Springer-Verlag, New York (1979). 